\title{
MINIMUM COVERS FOR ARCS OF CONSTANT LENGTH
}

\author{
BY GEORGE POOLE AND JOHN GERRIETS
}

Communicated by Mary Ellen Rudin, September 22, 1972

Recently Gerriets [1] showed that a certain convex closed region with area less than $0.3214 L^{2}$ covers any arc of length $L$. This is an improvement to Wetzel's results [3] on the famous and elusive "Worm Problem" of Leo Moser [2]: What is the (convex) region of smallest area which will accommodate every arc of length $L$ ? Wetzel showed that a certain truncated sector with area less than $0.34423 L^{2}$ covers all arcs of length $L$. By slightly modifying the region considered by Gerriets, we obtain a region with area less than $0.2887 L^{2}$ which covers any arc of length $L$.

THEOREM. The closed region whose boundary is a rhombus with major diagonal $L$ and minor diagonal $L / 3^{1 / 2}$ covers any arc of length $L$.

Herein we give a sketch of the proof. Details and other results will appear elsewhere. Let $P Q$ denote an arc of length $L$ and with center $C$ whose two subarcs are $P C$ and $C Q$. "Slide" the arc $P Q$ along $B E$ toward $B$ so that $C$ is always incident with $B E$ and $P Q$ becomes "tangent" to $A B$ or $B D$ (see the figure below) at the points $X$ or $Y$. It is possible that all such

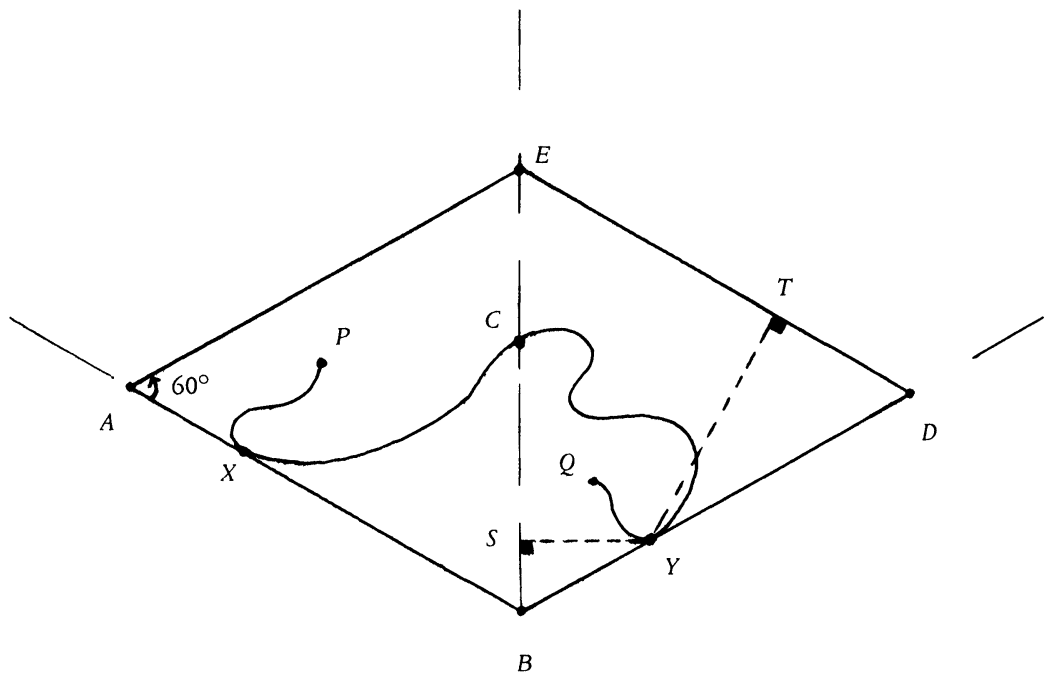

orientations of $P Q$ by rotation allow only one of the arcs $P C$ or $C Q$ to be

AMS (MOS) subject classifications (1970). Primary 52A45, 52A40.

Key words and phrases. Arcs, convex regions. 
tangent to the angle $A B D$ with all other points of the arc $P Q$ lying on or above the angle. Assume for the present, however, that there are two tangent points $X, Y$ on $P C, C Q$ which lie on the segments $A B$ and $B D$, respectively. Construct the segments $S Y$ and $Y T$ perpendicular to $B E$ and $D E$, respectively (the case when a point between $C$ and $Y$ meets $D E$ is handled in a similar way to the case under discussion). If $C Q$ agrees with the segments $S Y$ and $Y T$, then the length of $C Q$ is exactly $L / 2$ and, hence, is covered by the region $R$ described in the Theorem. If $C Q$ does not agree with $S Y$ and $Y T$, then in order for $C Q$ to get to the boundary, its length must exceed the length of $S Y T$ (which is $L / 2$ ), an impossibility. So $C Q$ is covered by $R$ and, similarly, $P C$ is also covered. Symmetry of $R$ dispenses with the case that $P Q$ has only one subarc tangent to $A B D$.

It can be shown that the region $R$ can be truncated to obtain a region with area less than $0.2861 L^{2}$ which covers any arc of length $L$.

The authors wish to thank Professor John E. Wetzel for sharing the results in [3] prior to publication.

\section{REFERENCES}

1. John Gerriets and George Poole, Covers accommodating curves of constant length (submitted).

2. Leo Moser, Poorly formulated unsolved problems of combinatorial geometry (mimeographed).

3. John E. Wetzel, Sectorial covers for curves of constant length, Canad. Math. Bull. (to appear).

Department of Mathematics, Kansas State Teachers College, Emporia, Kansas 66801 